Supporting Information

\title{
Dynamic Ordering in High- $\chi$ Block Copolymer Lamellae Based on Cross-Sectional Orientational Alignment
}

Ryuichi Nakatani, ${ }^{\dagger}$ Alvin Chandra,$^{\dagger}$ Takumi Uchiyama, Yuta Nabae, and Teruaki

Hayakawa*

Department of Materials Science and Engineering, School of Materials and Chemical

Technology, Tokyo Institute of Technology, 2-12-1-S8-36 Ookayama, Meguro-ku, Tokyo

152-8552, Japan.

*e-mail: hayakawa.t.ac@m.titech.ac.jp

\section{List of contents:}

Figure S1. S2. S3. S4. S5.

Programming Codes 


\section{Calculation of local orientation angle and color map preparation.}

The self-assembled structures of block copolymers (BCPs) and their orientation on thin films arise from the macroscopic orientation of the polymer chains which are considered to be similar to liquid crystalline molecules. Therefore, the order parameter for microdomains in two dimensions can be defined as below, where $\theta$ is the local orientation of the microdomains.

$$
\psi=\psi_{0} \exp (2 i \theta)
$$

To determine the local orientation angle $\theta$, a set of image processing programs was constructed by following a Matlab routine. Our programming algorithm is based on the procedure proposed by Harrison. ${ }^{1}$ Real space phase images were obtained from atomic force microscopy (AFM) measurements using standard silicon tips (OMCL-AC55TN, Olympus) with an average radius of $\sim 7 \mathrm{~nm}$ and scanning electron microscopy (SEM) for the cross-section. The AFM phase images were recorded using intermittent contact (AC) mode with a resolution of $0.98 \mathrm{~nm} / \mathrm{pixel}$ and were first converted to $q$ space images by fast Fourier transform (FFT). The average radial profiles were then produced from the integrated intensity at any given distance divided by the number of pixels. The jagged profiles were smoothed using a Gaussian function to pick the local maxima. After peakpicking, the frequencies roughly $20 \%$ above and below $q_{0}$, the dominant frequency, were eliminated. Through this process, high and low frequency noises such as salt-and-pepper and charging effects could be reduced. Inverse FFT was then performed to transform the filtered images to real space images. Each image intensity in the Fourier-filtered images 
were smoothed out by Gaussian-averaging each pixel with its neighbors to produce a welldefined two-dimensional gradient at all points as below, where $I(i, j)$ is the image intensity at location $(i, j)$.

$$
\begin{aligned}
& \nabla_{x}=I(i+1, j)-I(i, j) \\
& \nabla_{y}=I(i, j+1)-I(i, j)
\end{aligned}
$$

Two-dimensional gradient $\left(\nabla_{x}, \nabla_{y}\right)$ can also be expressed from (1) by equations:

$$
\begin{aligned}
& \nabla_{x}=\psi_{0} \cos \theta \\
& \nabla_{y}=\psi_{0} \sin \theta
\end{aligned}
$$

Therefore, $\theta$ can be calculated:

$$
2 \theta=\arctan \left(\frac{\psi_{0}^{2} \sin 2 \theta}{\psi_{0}^{2} \cos 2 \theta}\right)=\arctan \frac{2 \nabla_{x} \nabla_{y}}{\nabla_{x}^{2}-\nabla_{y}^{2}}
$$

The value of sigma for Gaussian-averaging was calculated as below, where $L_{0}$ is the periodic length of the microdomains.

$$
\begin{gathered}
\sigma=\left(\frac{L_{0}-1}{2}\right)^{1 / 2} \\
L_{0}=2 \pi / q_{0}
\end{gathered}
$$

After a series of processing shown above, the local orientation angle $\theta$ at all points in the image can be visualized as a false-color map. To reduce the effects from roughness of the microdomains, binarization and thinning process were included after Gaussian-averaging. A threshold was set to be equal to the average pixel intensity in the image after averaging. 
The processed images and obtained profiles at each step are summarized in Figure S1 for the surface and Figure $\mathbf{S 2}$ for the cross-section. 
(a)

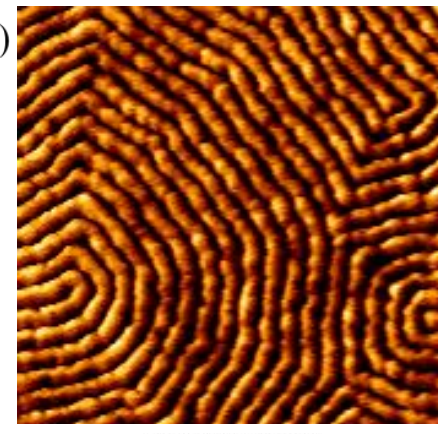

(d)

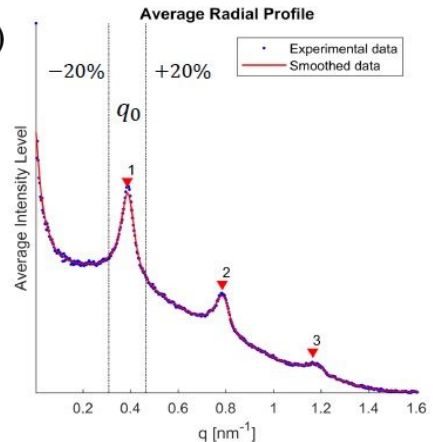

(g)

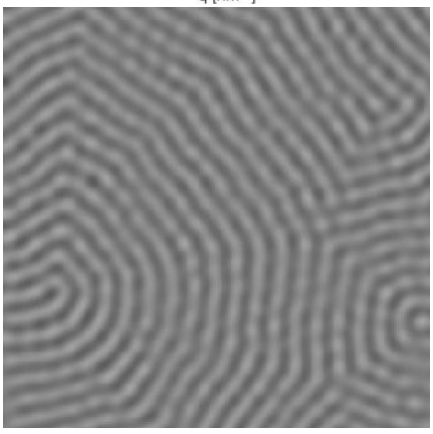

(j)

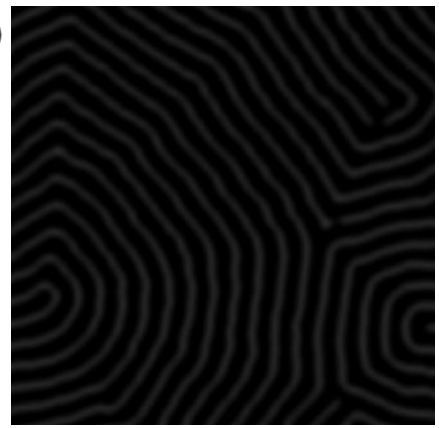

(b)

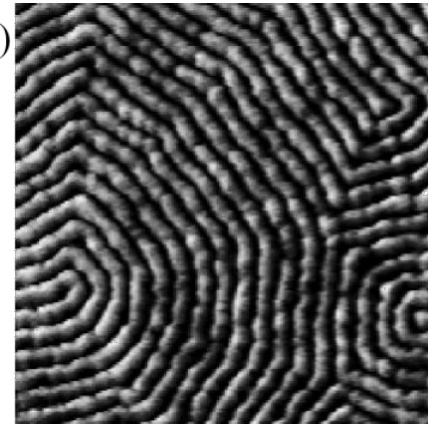

(e)

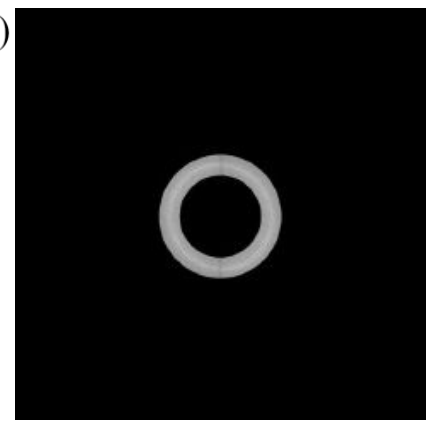

(h)

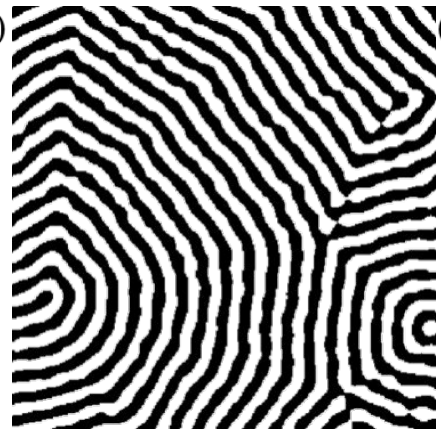

(k)

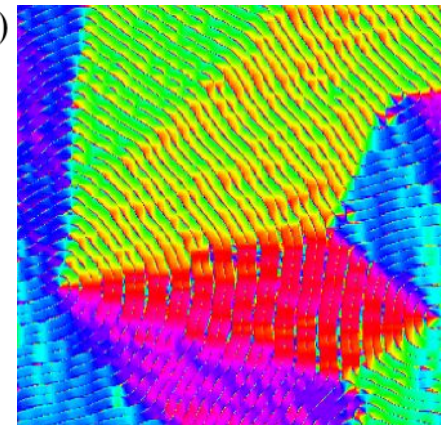

(c)

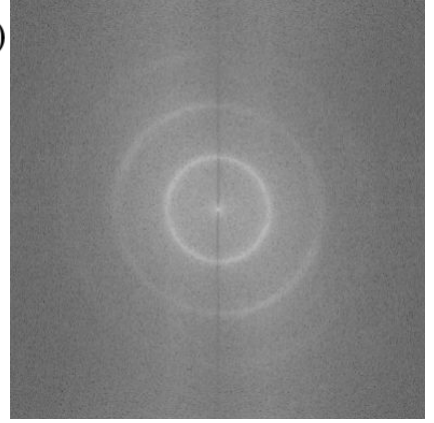

(f)

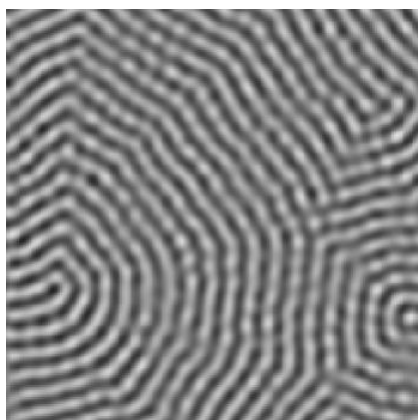

i)

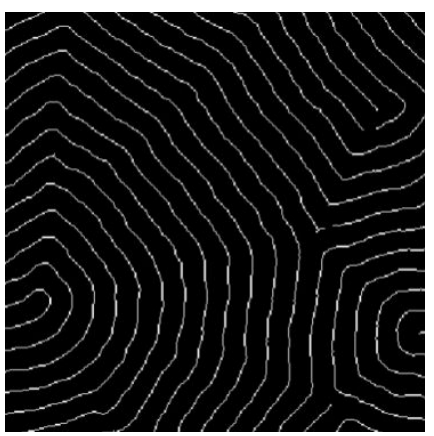

$\theta$ : orientation angle
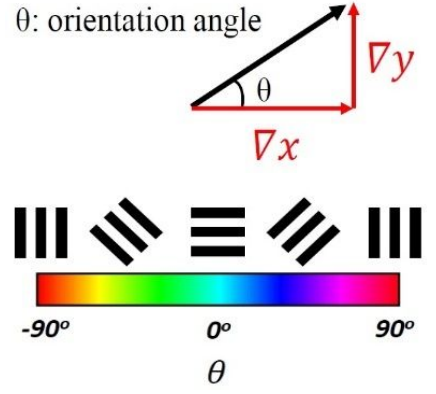

Figure S1. (a) AFM image before processing. (b) 8-bit gray scale image. (c) FFT image.

(d) average radial profile. (e) band-pass filtered image. (f) inverse FFT image. (g) smoothed image by Gaussian averaging. (h) binary image. (i) skeletonized image. (j) smoothed image of (i) by Gaussian averaging. (k) orientation color map. 
(a)

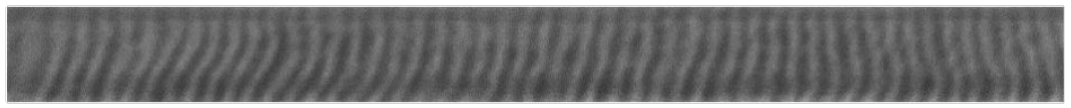

(b)

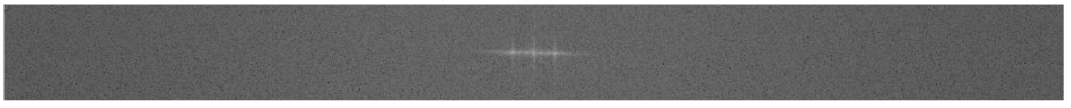

(c)

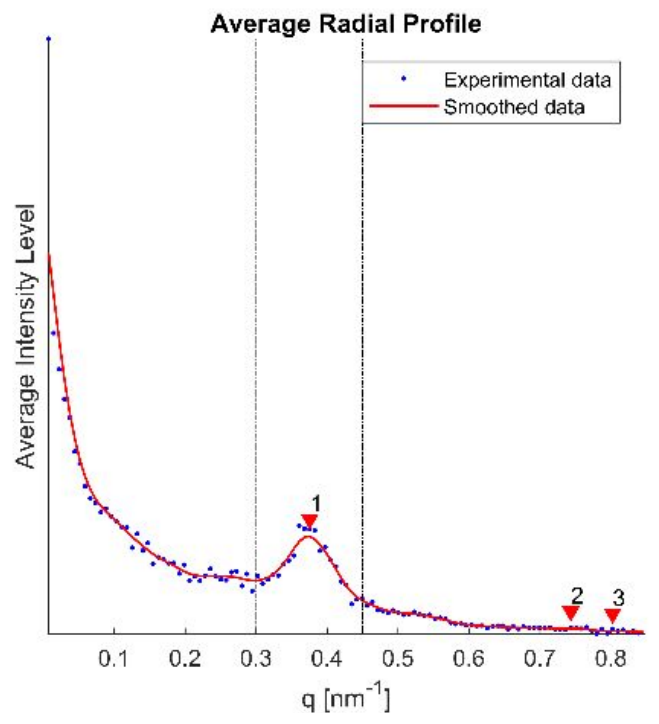

(d)

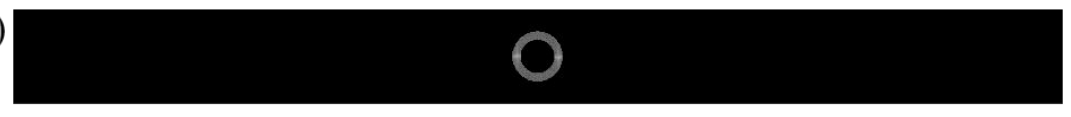

(e)

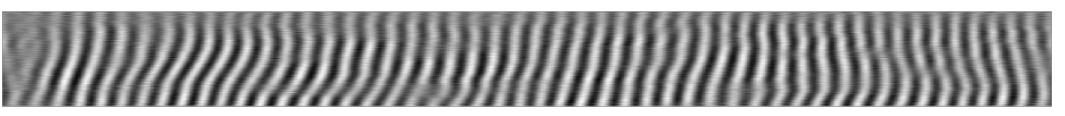

(f)

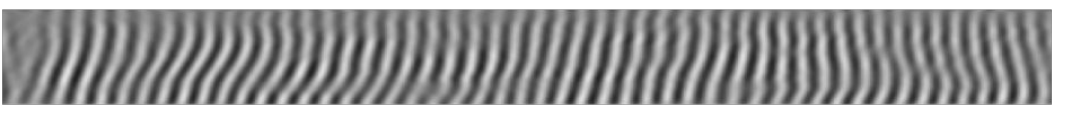

(g)
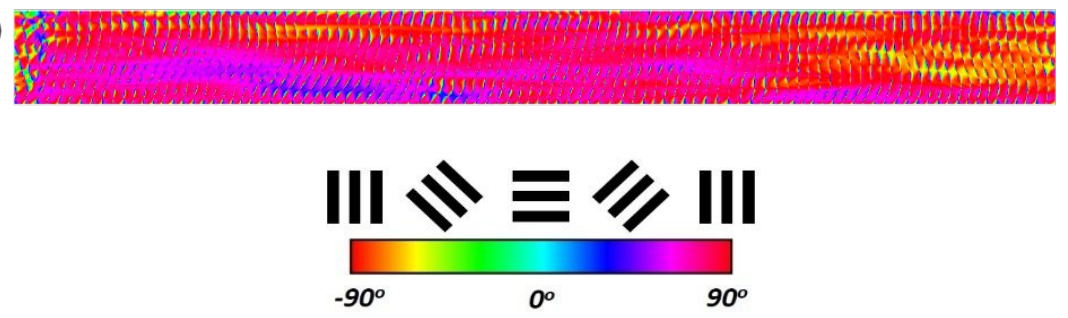

Figure S2. (a) Cross-sectional SEM image before processing. (b) FFT image. (c) average radial profile. (d) band-pass filtered image. (e) inverse FFT image. (f) smoothed image by Gaussian averaging. (g) orientation color map. 


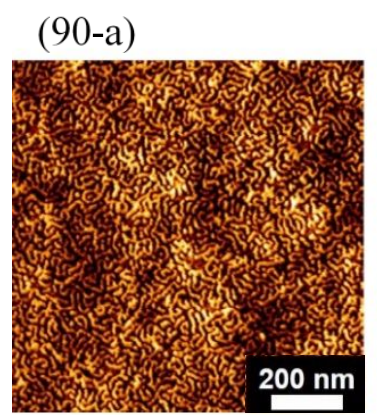

(90-e)

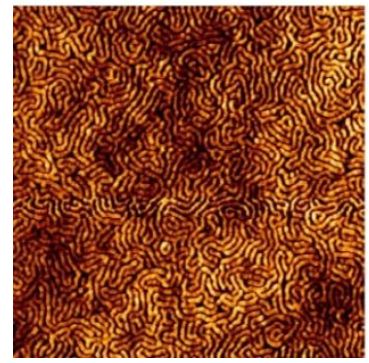

(90-i)

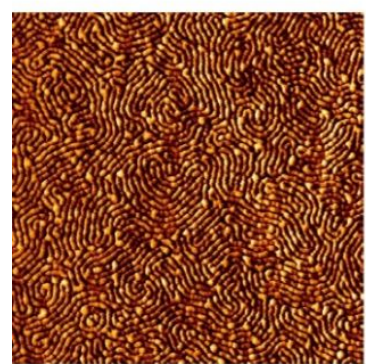

(90-m) (90-b)

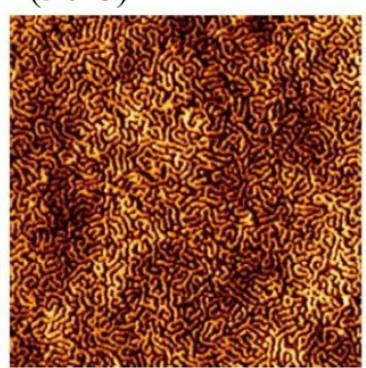

(90-c)

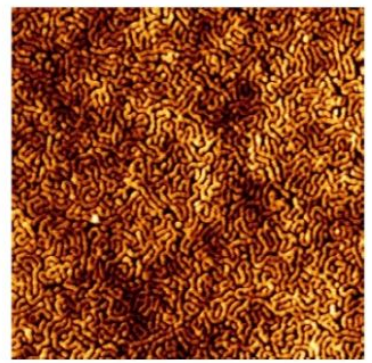

(90-f)

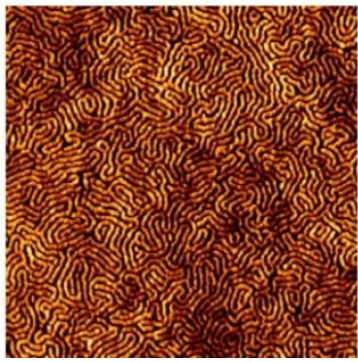

(90-j)

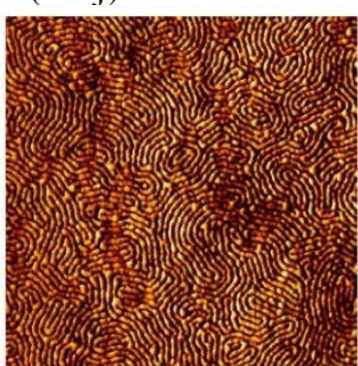

(90-g)

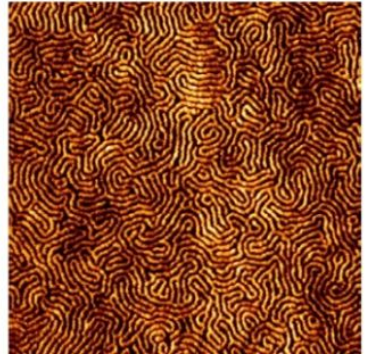

(90-k)

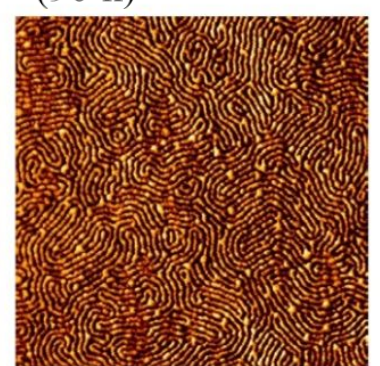

(90-d)

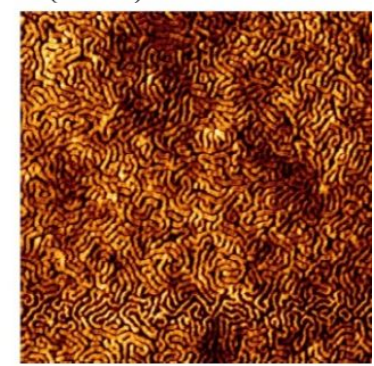

(90-h)

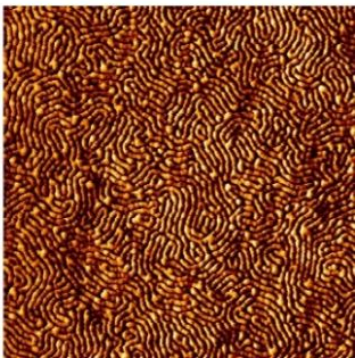

(90-1)

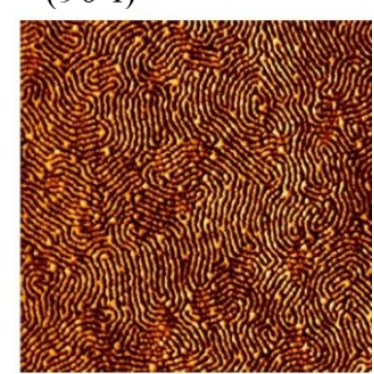

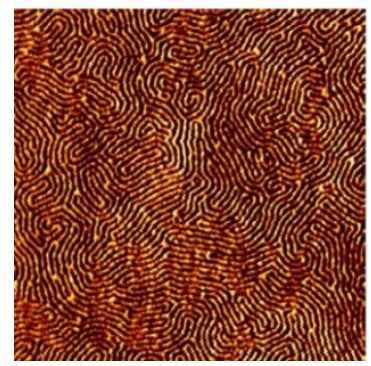



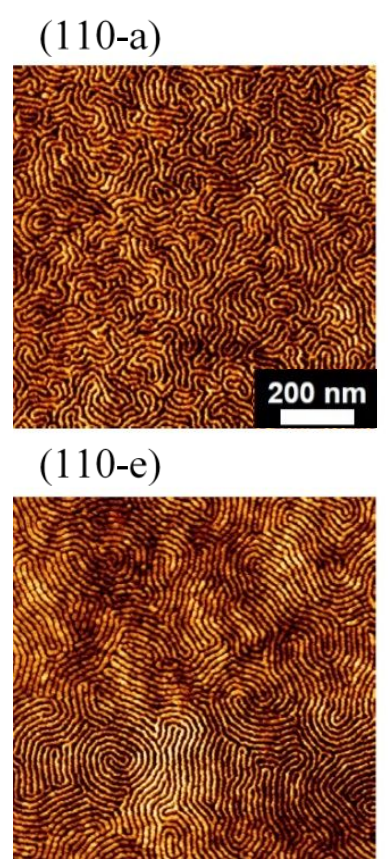

(110-i)

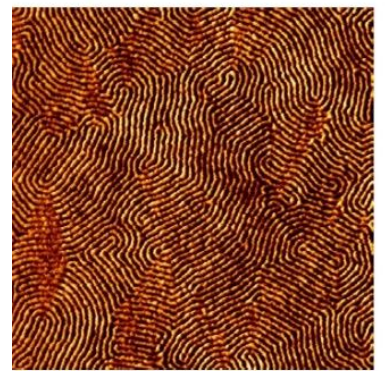

(110-m)

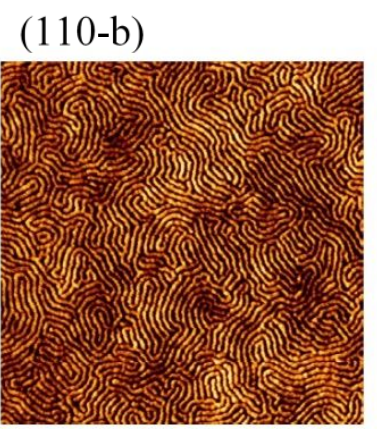

(110-f)

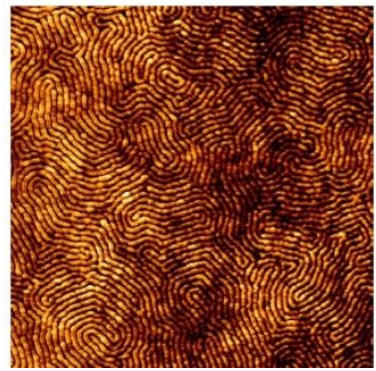

(110-j)

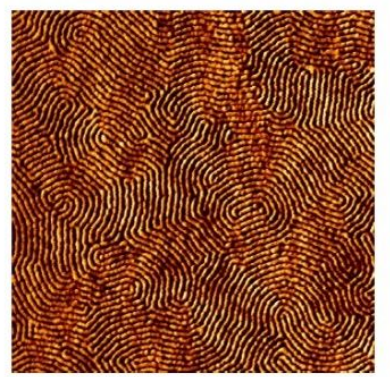

(110-c)

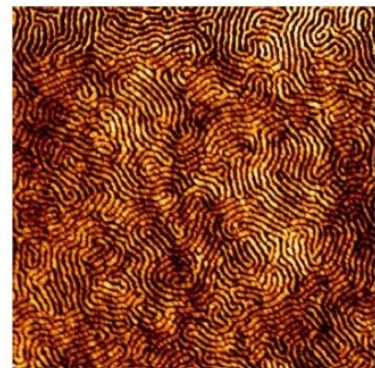

(110-g)

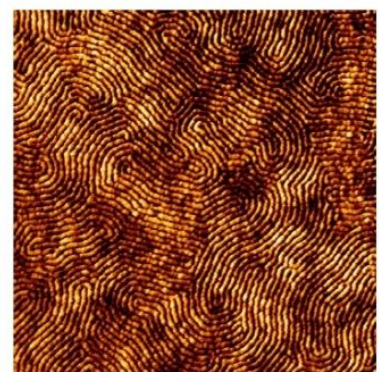

(110-k)

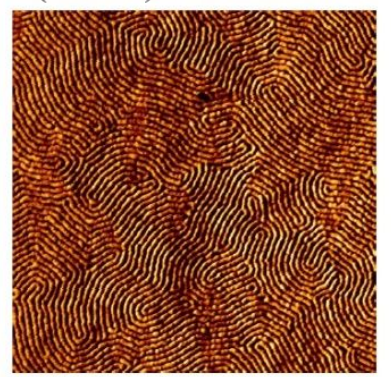

(110-d)

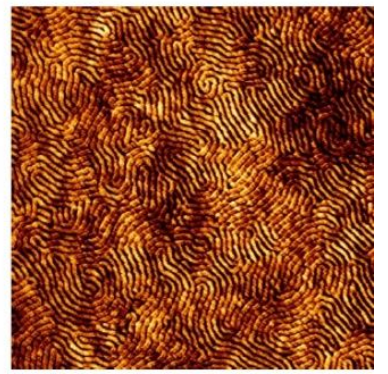

(110-h)

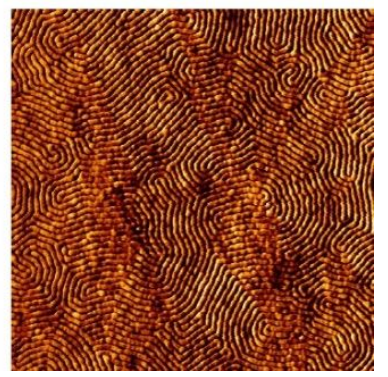

(110-1)

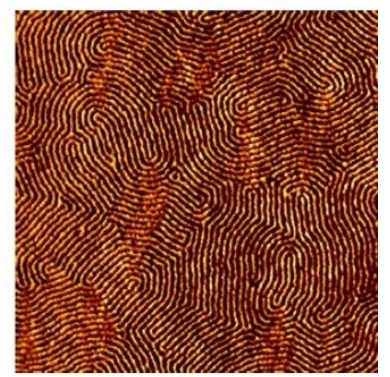

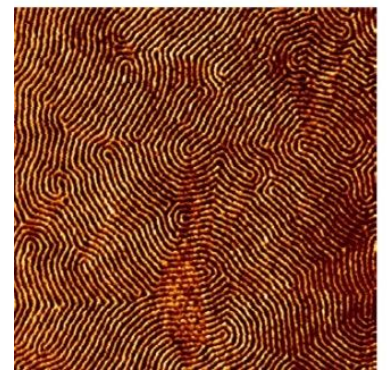



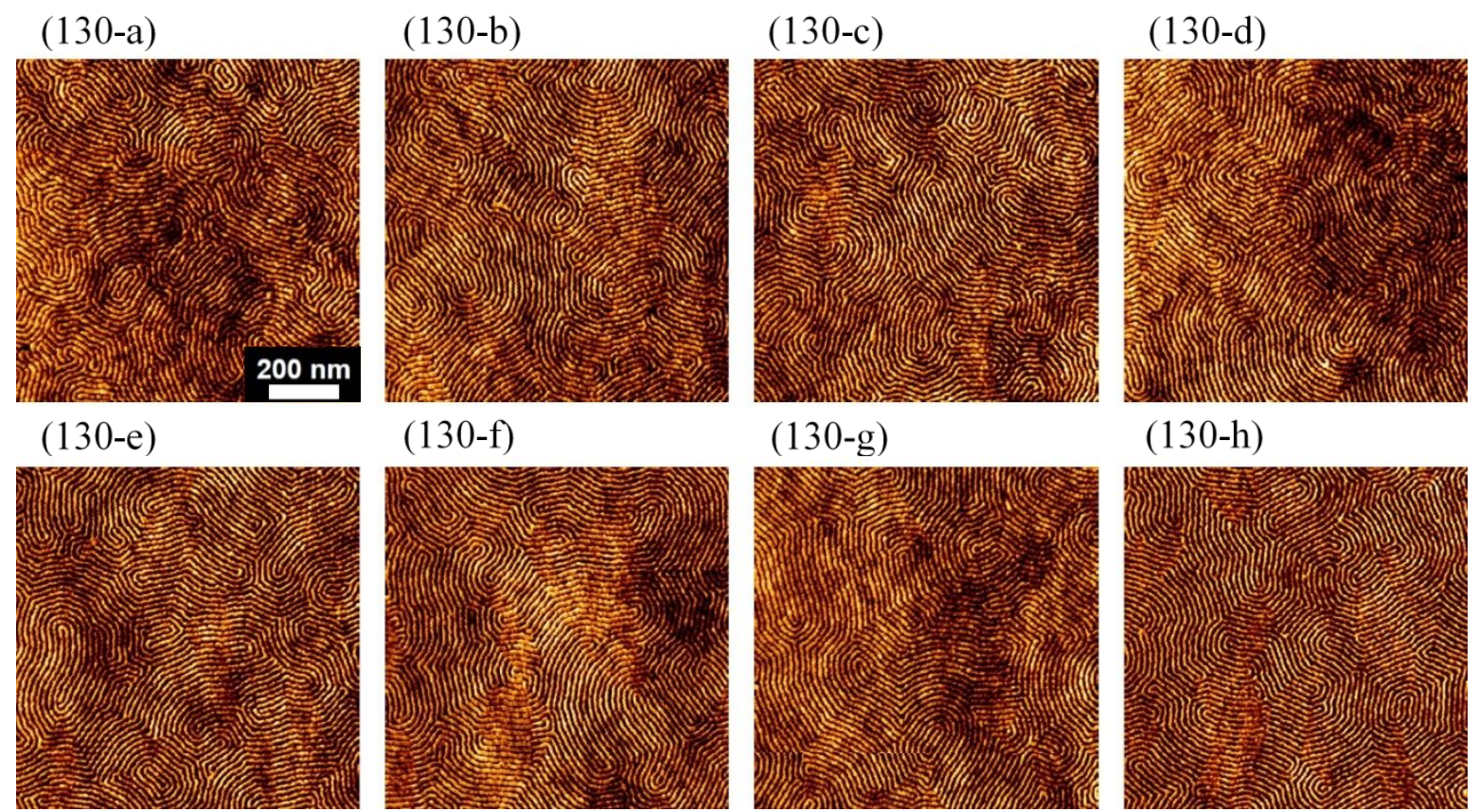

(130-f)

(130-g)

(130-h)
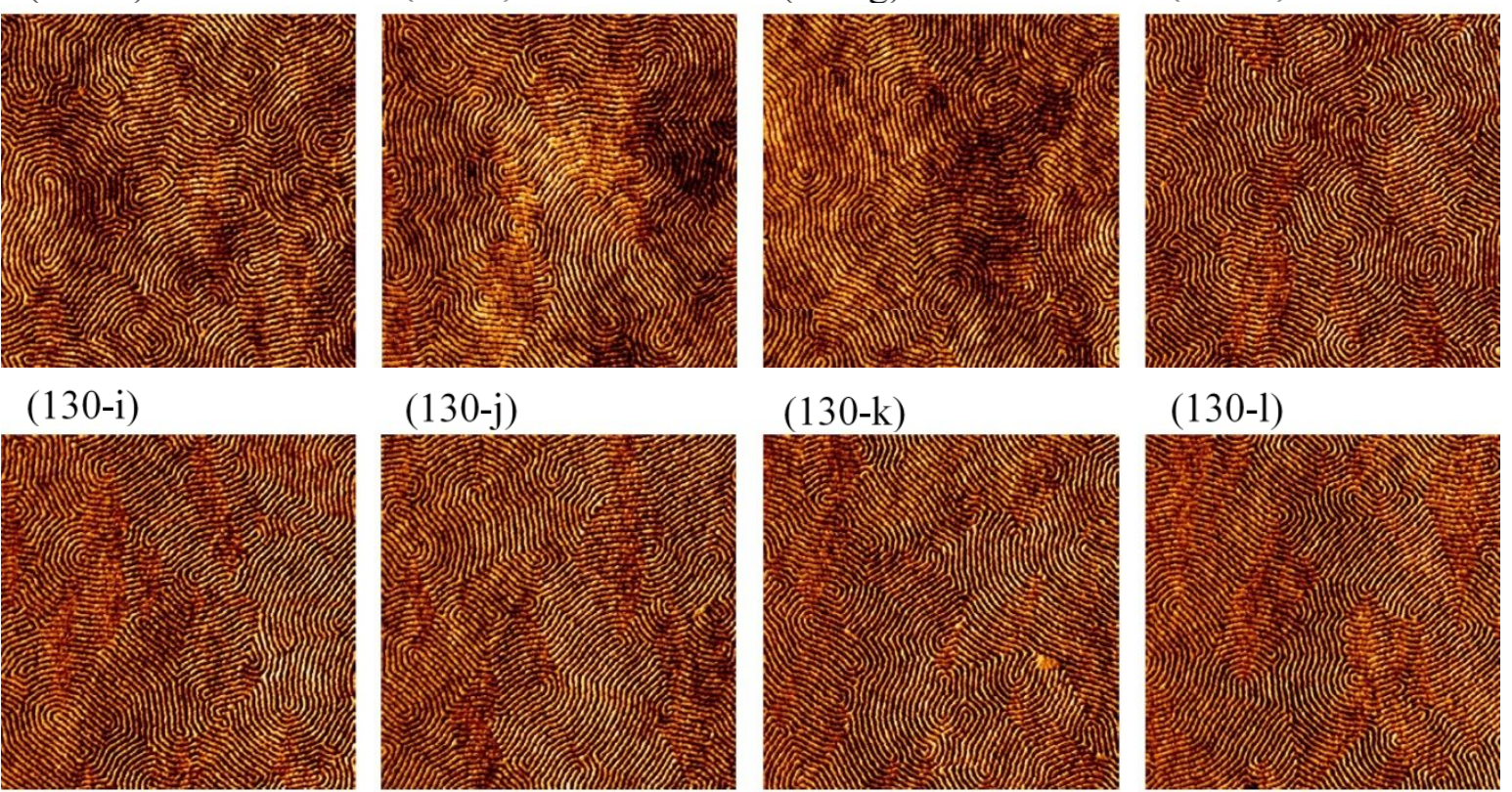

(130-j)

(130-k)

(130-1)
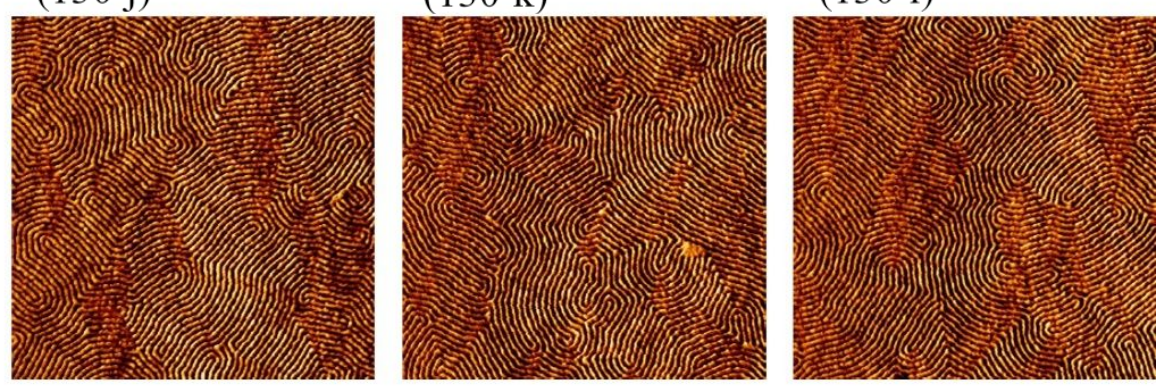

\section{(130-m)}



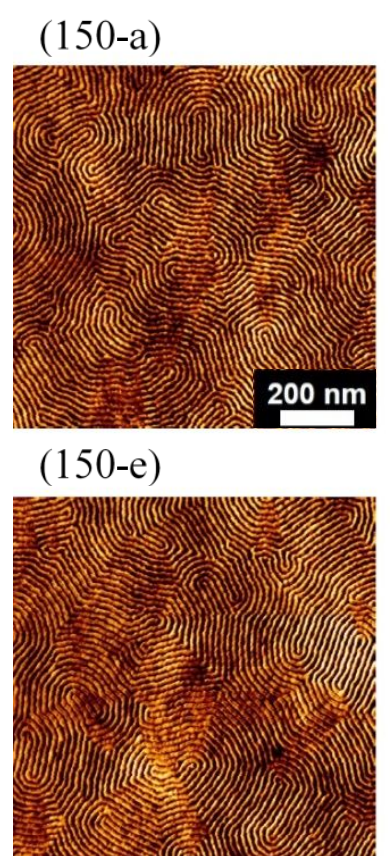

(150-i)

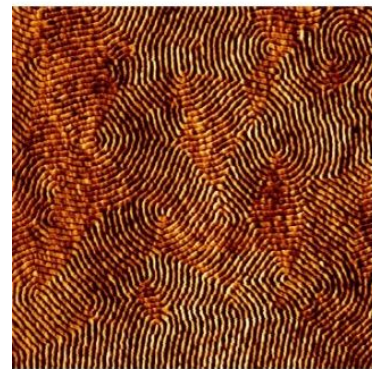

(150-m) (150-b)

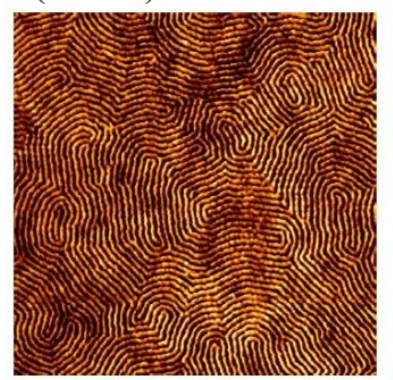

(150-f)

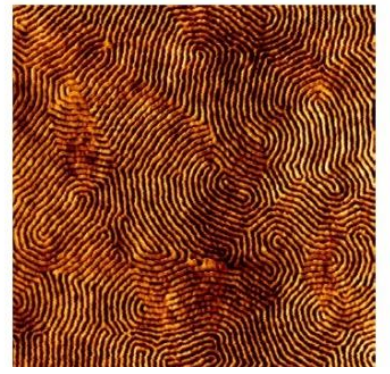

(150-j)

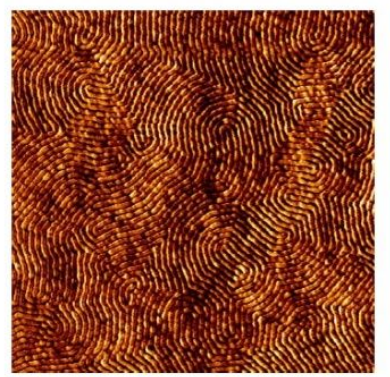

(150-c)

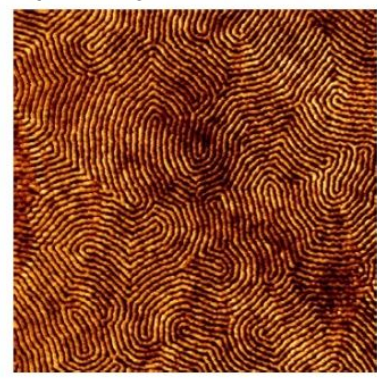

(150-g)

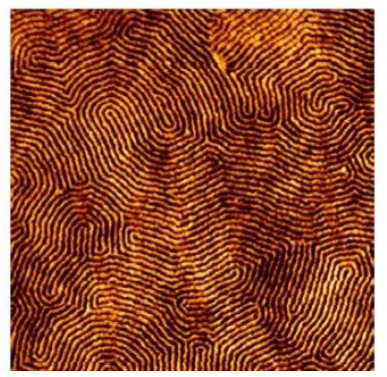

(150-k)

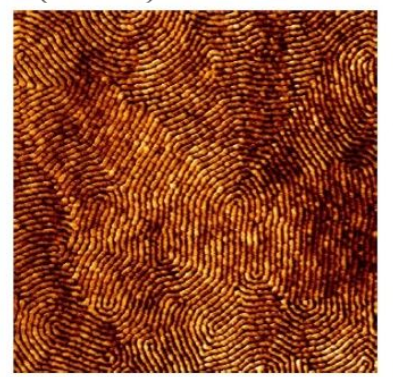

(150-d)

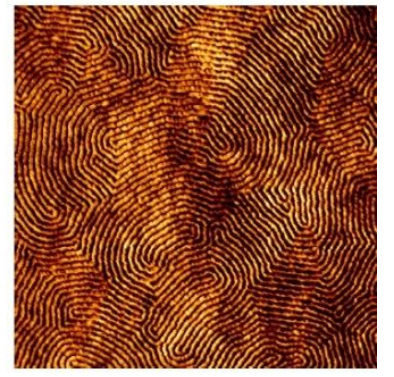

(150-h)

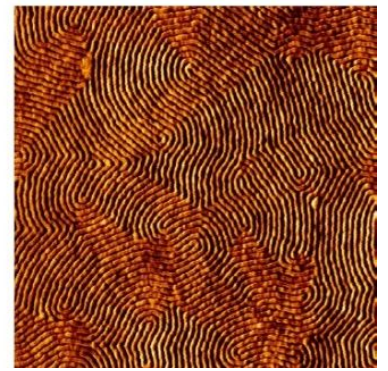

(150-1)

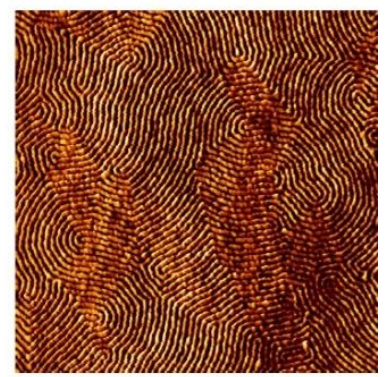

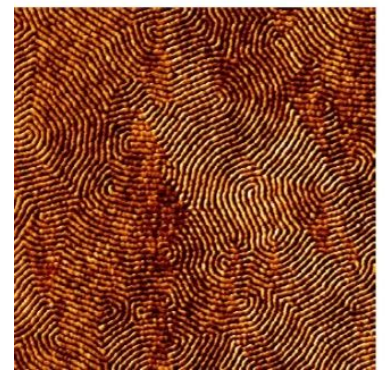



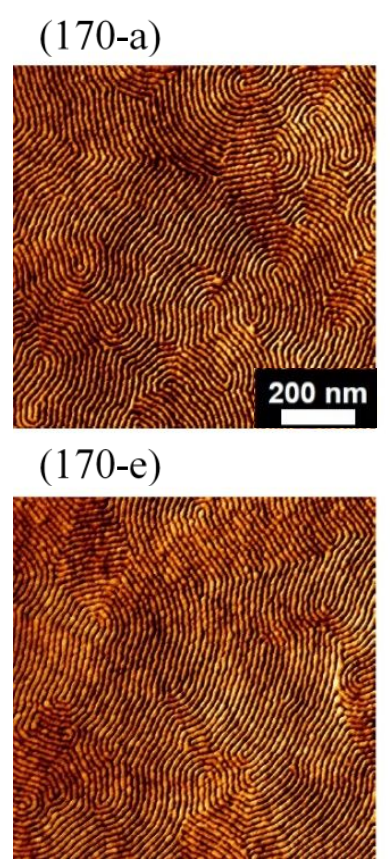

(170-i)

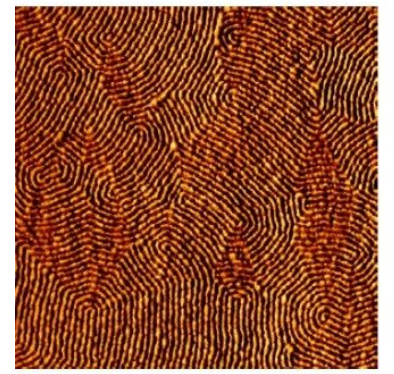

(170-m)

$$
\text { (170-b) }
$$

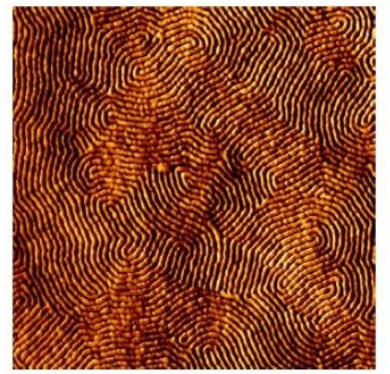

(170-f)

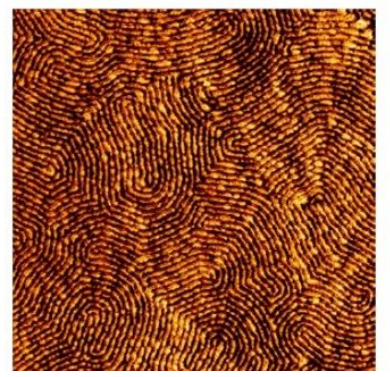

(170-j)

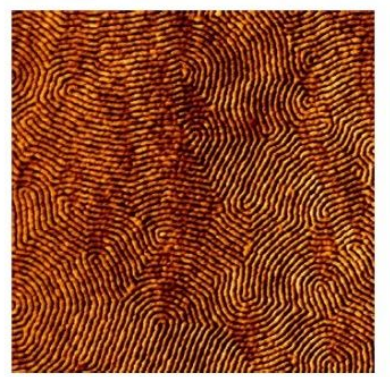

(170-c)

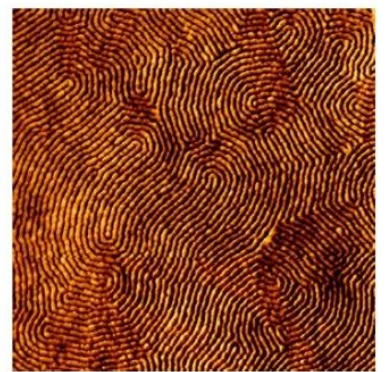

(170-g)

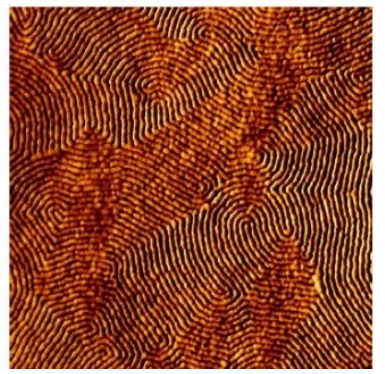

(170-k)

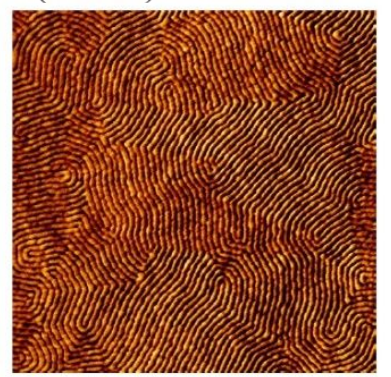

(170-d)

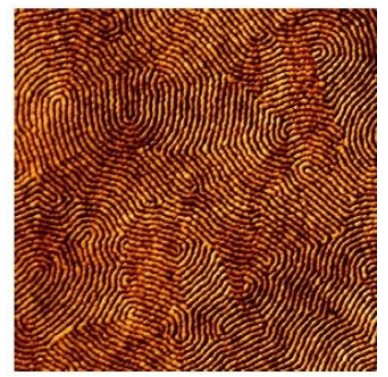

(170-h)

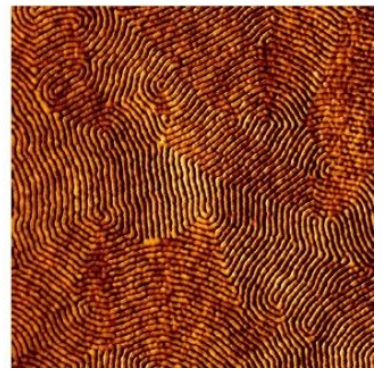

(170-1)

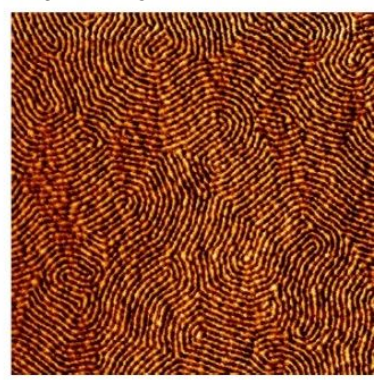

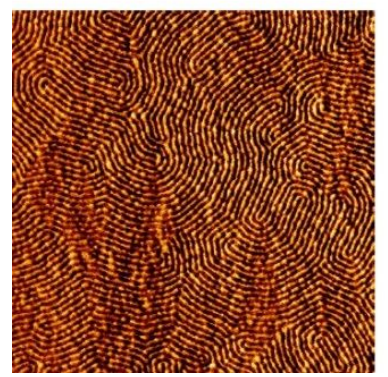



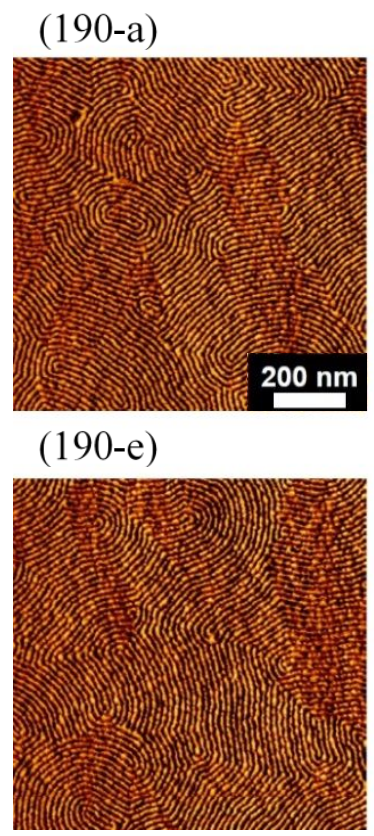

(190-i)

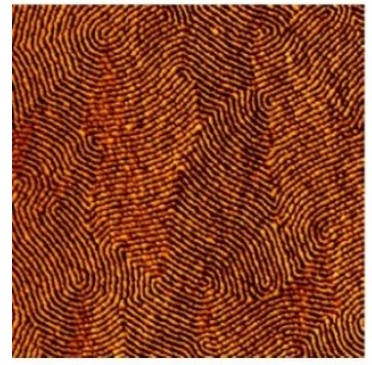

(190-m) (190-b)

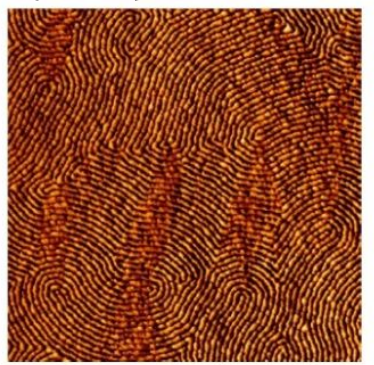

(190-f)

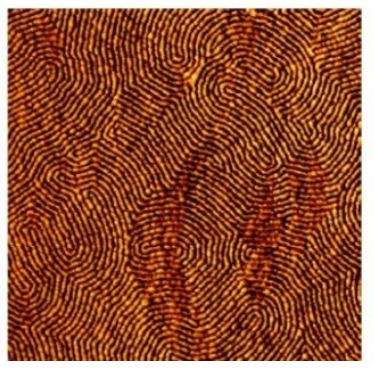

$(190-\mathrm{j})$

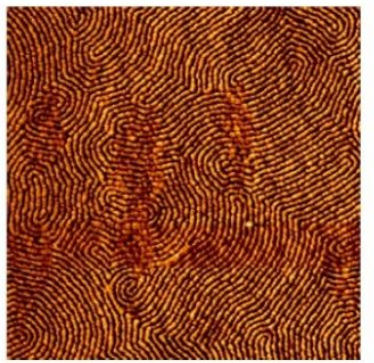

(190-c)

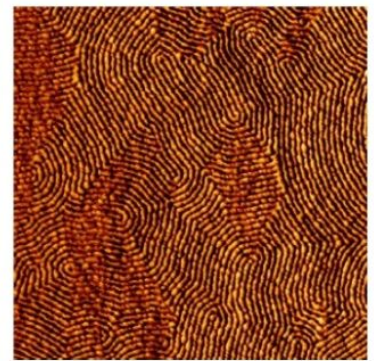

(190-g)

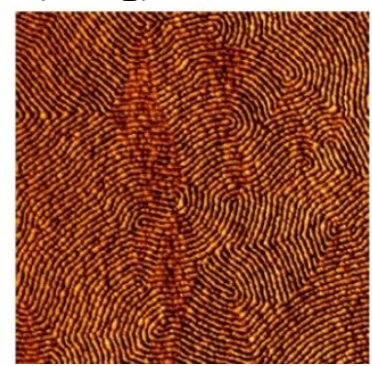

$(190-k)$

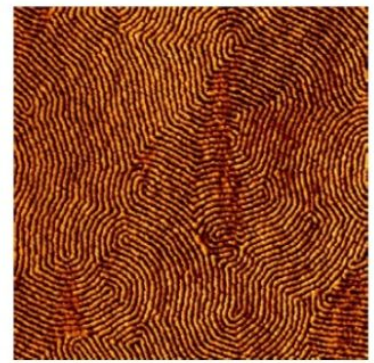

$(190-d)$

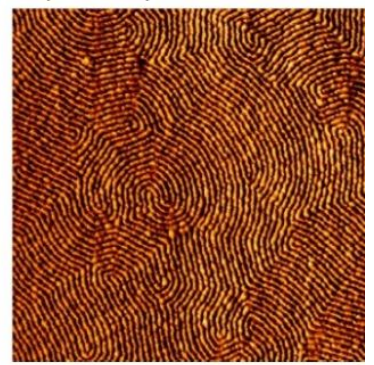

(190-h)

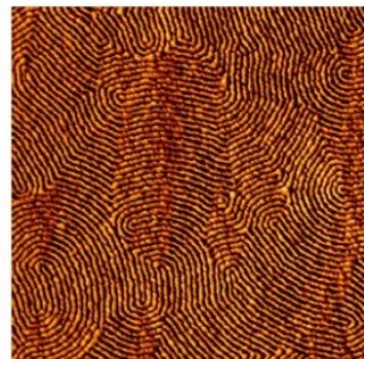

(190-1)

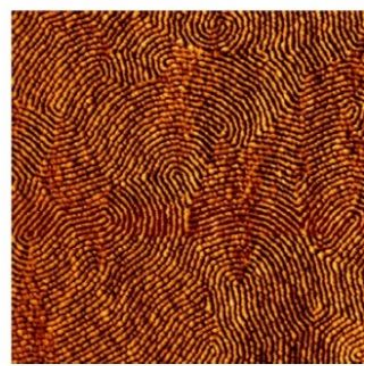

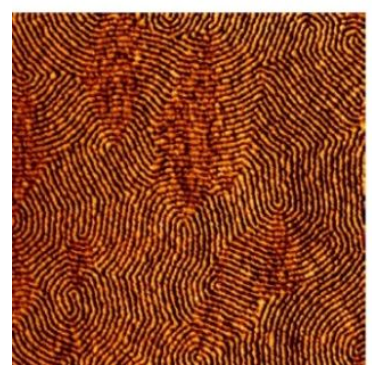

Figure S3. AFM phase images of PMAPOSS 6 - $b$-PTFEMA ${ }_{80}$ thin films thermally annealed at various temperatures and for various times. The numbers in the caption indicate the temperature, while the letters indicate the annealing times. (a) $1 \mathrm{~min}$, (b) $3 \mathrm{~min}$, (c) $5 \mathrm{~min}$, (d) $10 \mathrm{~min}$, (e) $30 \mathrm{~min}$, (f) $1 \mathrm{~h}$, (g) $2 \mathrm{~h}$, (h) $4 \mathrm{~h}$, (i) $8 \mathrm{~h}$, (j) $12 \mathrm{~h}$, (k) $16 \mathrm{~h}$, (l) $20 \mathrm{~h}$ and (m) 24 $\mathrm{h}$. The size of every image is $1 \times 1 \mu \mathrm{m}$. 

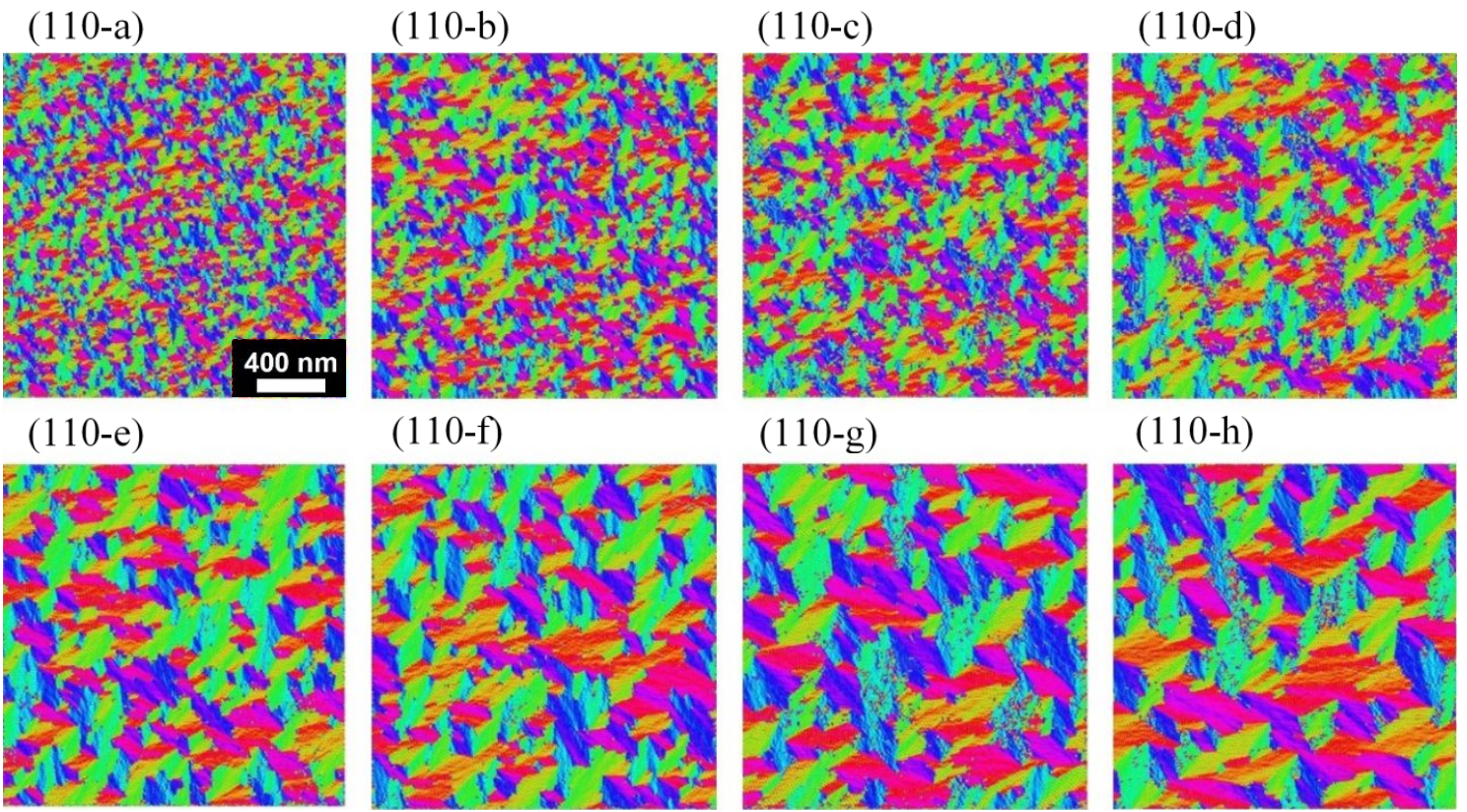

$(110-\mathrm{f})$

$(110-\mathrm{g})$

$(110-\mathrm{h})$
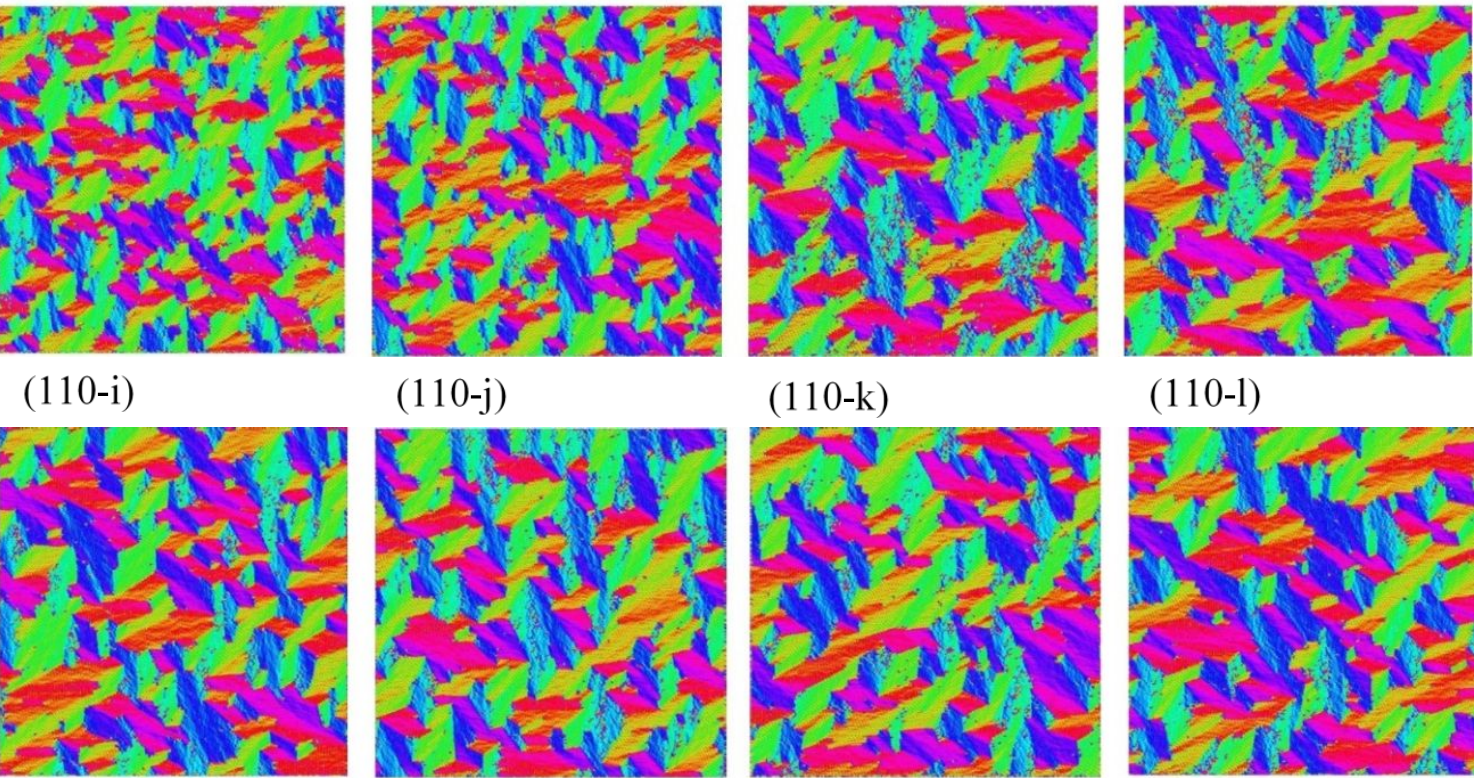

$(110-\mathrm{j})$

$(110-\mathrm{k})$

$(110-1)$
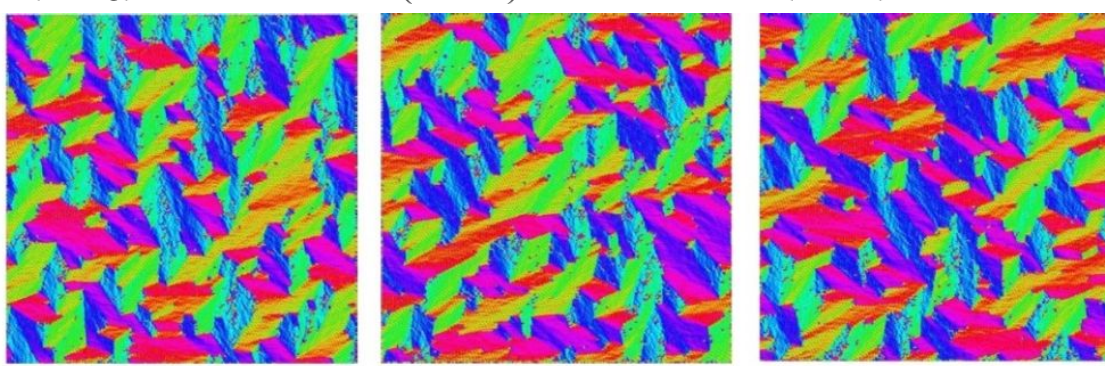

$(110-\mathrm{m})$

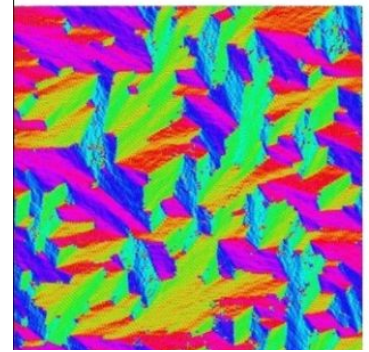

Figure S4. Orientational color maps of PMAPOSS $_{6}-b-\mathrm{PTFEMA}_{80}$ thin films thermally annealed at $110{ }^{\circ} \mathrm{C}$ for various times. The numbers in the caption indicate the annealing temperatures, while the letters indicate the annealing times. (a) $1 \mathrm{~min}$, (b) $3 \mathrm{~min}$, (c) $5 \mathrm{~min}$, (d) $10 \mathrm{~min}$, (e) $30 \mathrm{~min}$, (f) $1 \mathrm{~h}$, (g) $2 \mathrm{~h}$, (h) $4 \mathrm{~h}$, (i) $8 \mathrm{~h}$, (j) $12 \mathrm{~h}$, (k) $16 \mathrm{~h}$, (l) $20 \mathrm{~h}$ and (m) 24 $\mathrm{h}$. The size of every image is $2 \times 2 \mu \mathrm{m}$. 
(a)

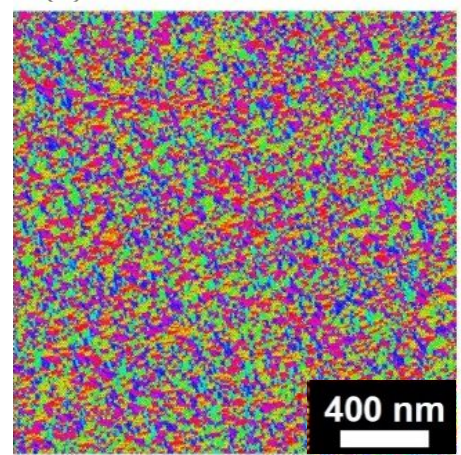

(d)

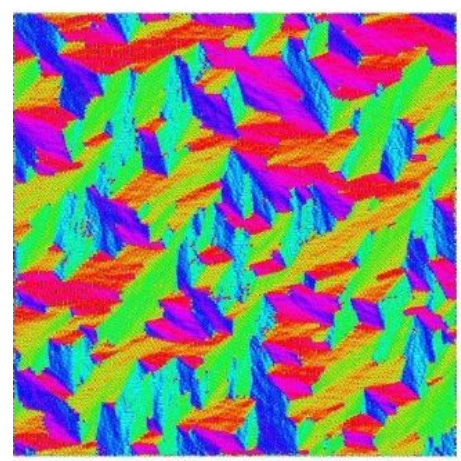

(b)

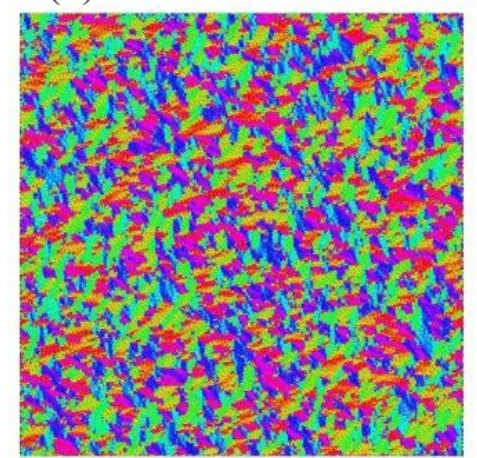

(e)

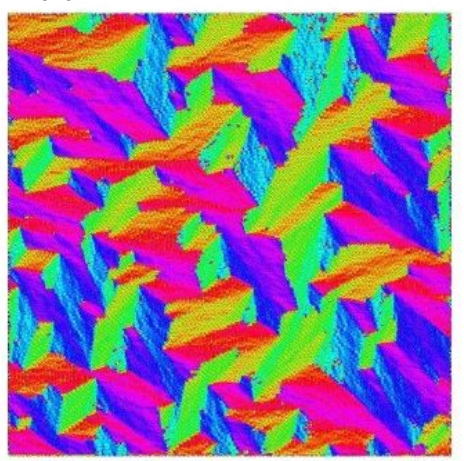

(c)

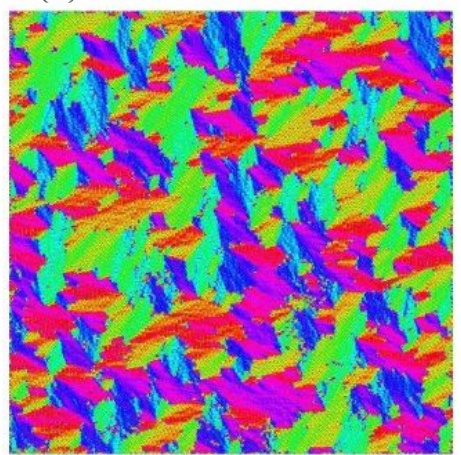

(f)

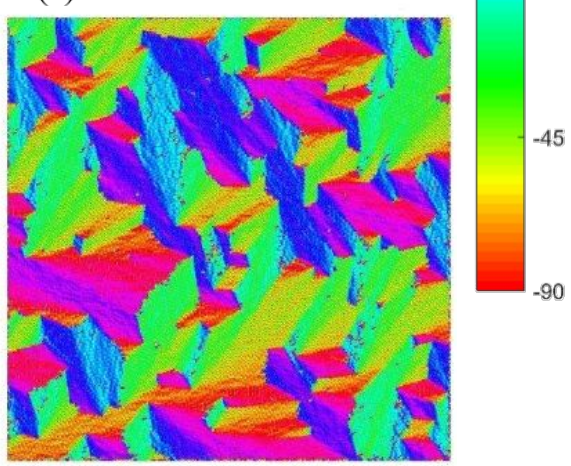

Figure S5. Orientational color maps of PMAPOSS $_{6}-b$-PTFEMA 80 thermally annealed at a variety of temperatures for $1 \mathrm{~min}$. (a) $90{ }^{\circ} \mathrm{C}$, (b) $110{ }^{\circ} \mathrm{C}$, (c) $130{ }^{\circ} \mathrm{C}$, (d) $150{ }^{\circ} \mathrm{C}$, (e) $170{ }^{\circ} \mathrm{C}$ and (f) $190^{\circ} \mathrm{C}$. The size of every image is $2 \times 2 \mu \mathrm{m}$. 


\section{Calculation of activation energy.}

In general, the correlation length $(\xi)$ is proportional to the average distance between separate defects that disrupt the ordering of the striped patterns. The time-dependent defect density $\rho(t)$ is given by: ${ }^{2}$

$$
\rho(t) \sim \xi^{-2}(t)
$$

By making a simplifying assumption where defects disrupting the orientational order all move at the same velocity $v(t)$, the measured correlation lengths can be related to the activation energies needed for defect annihilation. As the average distance between defects is proportional to $\xi(t)$, the defect velocity at a given time $t$ is proportional to the rate of change in correlation length:

$$
v(t) \sim \frac{d \xi(t)}{d t}=\phi \frac{\xi(t)}{t}
$$

where $\xi(t)$ is given by Eq. (13). When expressed in terms of the defect density $\rho$, Eq. (10) becomes:

$$
v(\rho) \sim \phi \xi_{0, T}^{1 / \phi} \rho^{(1-\phi / 2 \phi)}
$$

As the motion of defects in striped patterns is similar to that of vacancy or interstitial diffusion in solid crystals, a thermally activated behavior (i.e. $\left.v(t) \sim \exp \left(-E_{a} / k T\right)\right)$ can be expected. This activation energy $E_{a}$ can then be estimated by analyzing the defect speed $(v)$ at a constant defect density (i.e. at fixed defect separation distance) as a function of $T$ :

$$
\begin{gathered}
v\left(\rho=\rho_{\text {const }}, T\right)=\left.\phi \xi_{0, T}^{1 / \phi} \rho^{(1-\phi / 2 \phi)}\right|_{\rho=\rho_{\text {const }}}=C e^{-E_{a} / k T} \\
\ln \left(\xi_{0, T}^{1 / \phi}\right)=-\frac{E_{a}}{k T}+\text { const. }
\end{gathered}
$$


where $k$ is boltzmann constant.

\section{Programming Codes.}

The programming codes used are as follows.

\section{Calculation of correlation length.}




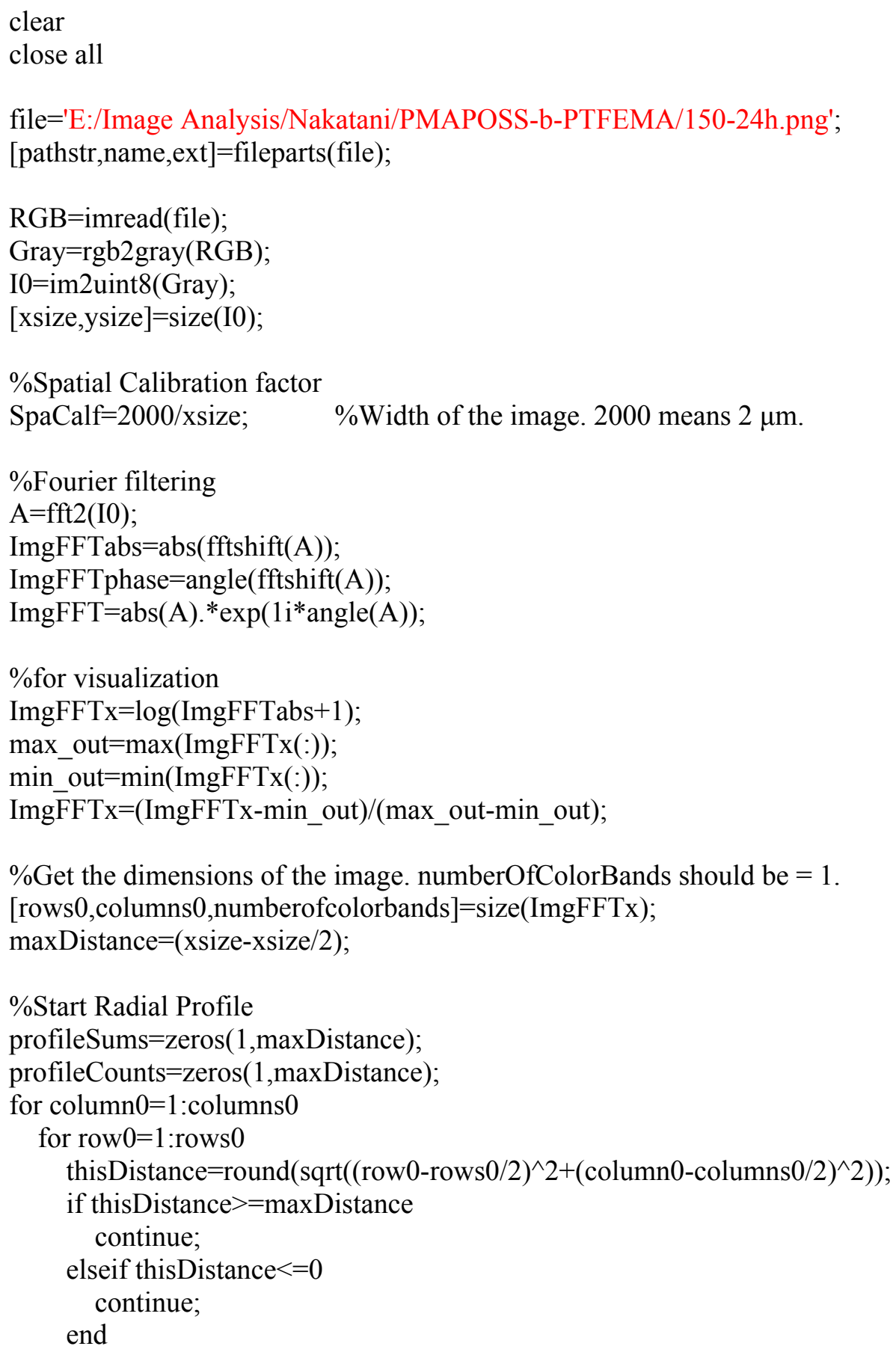




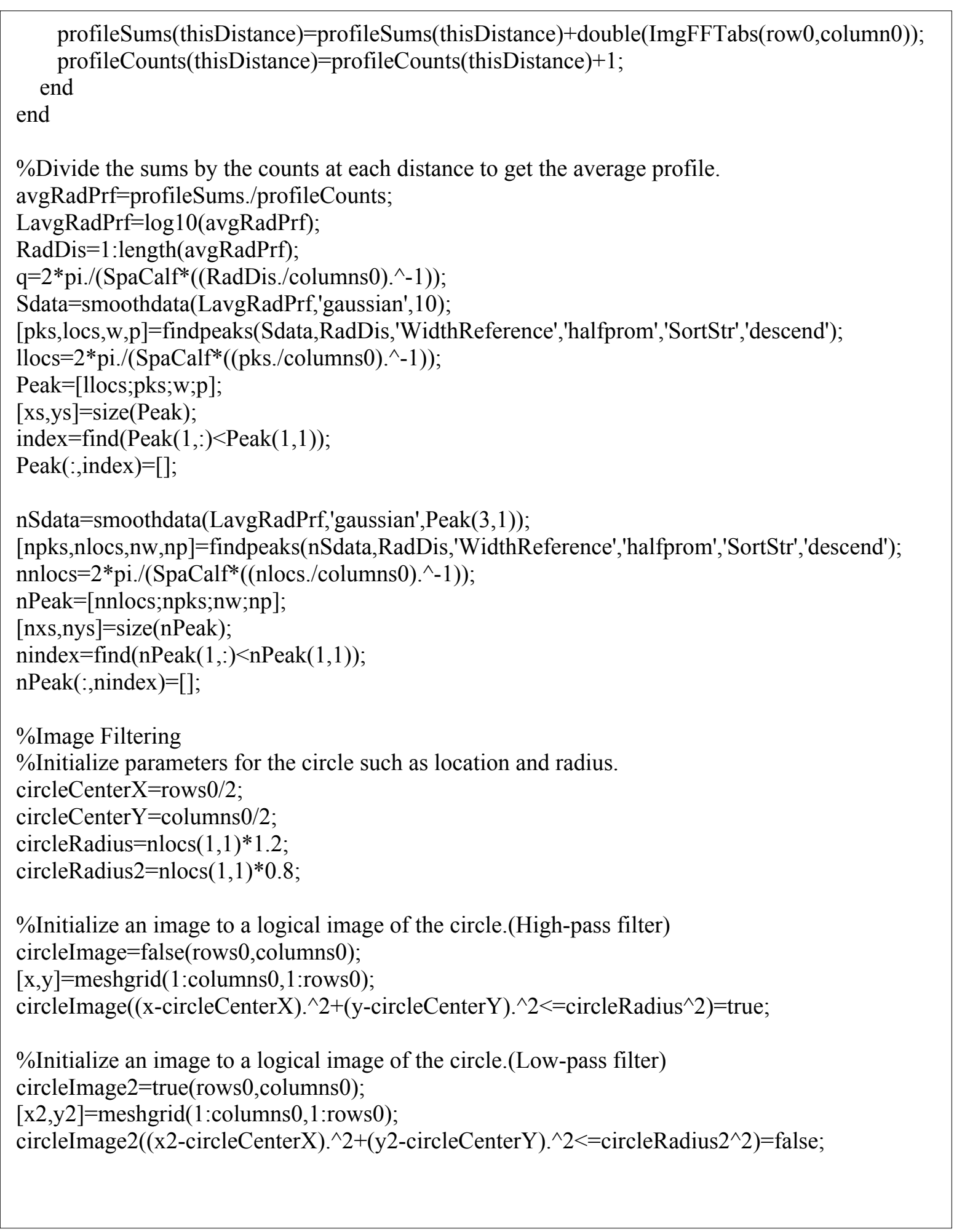




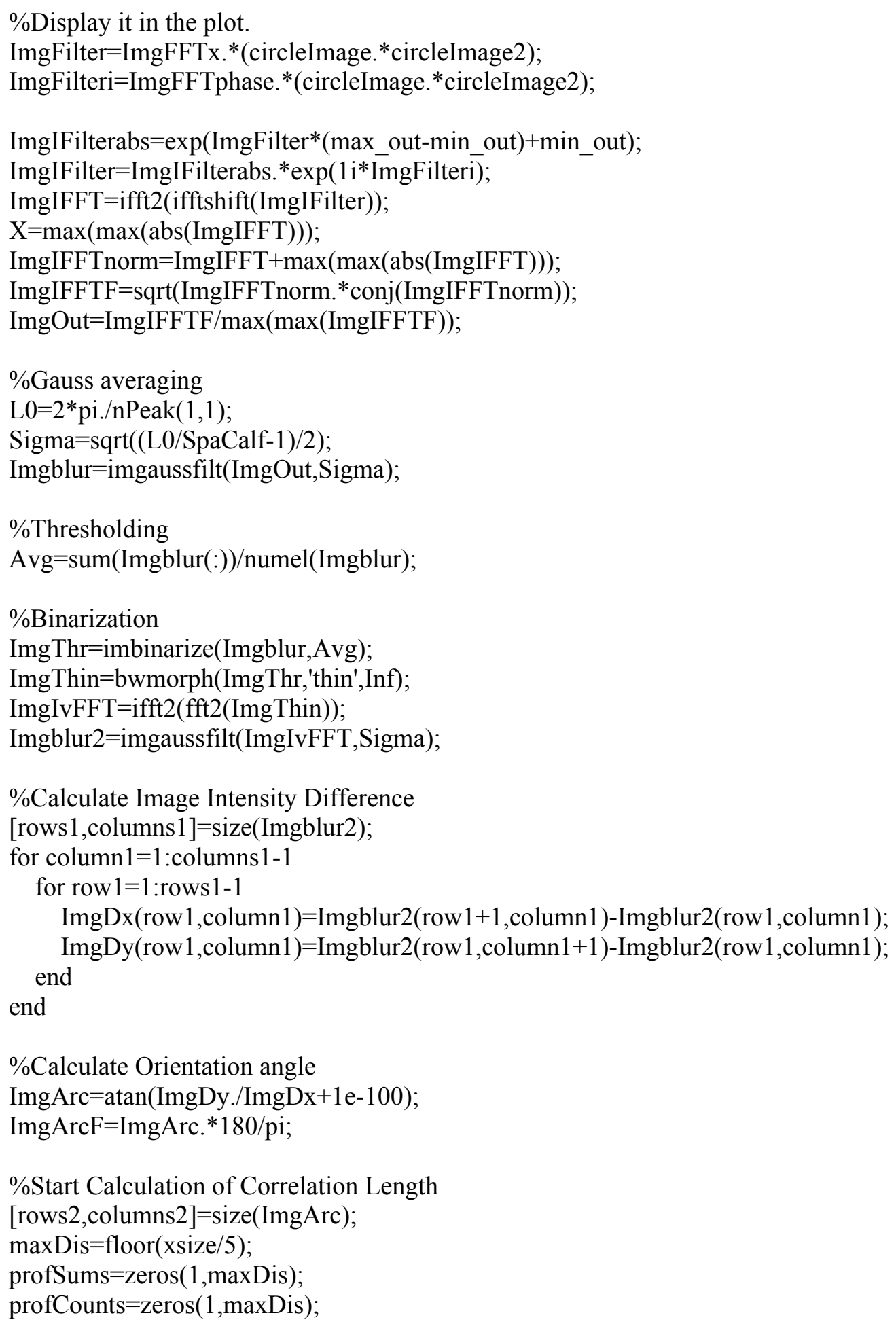




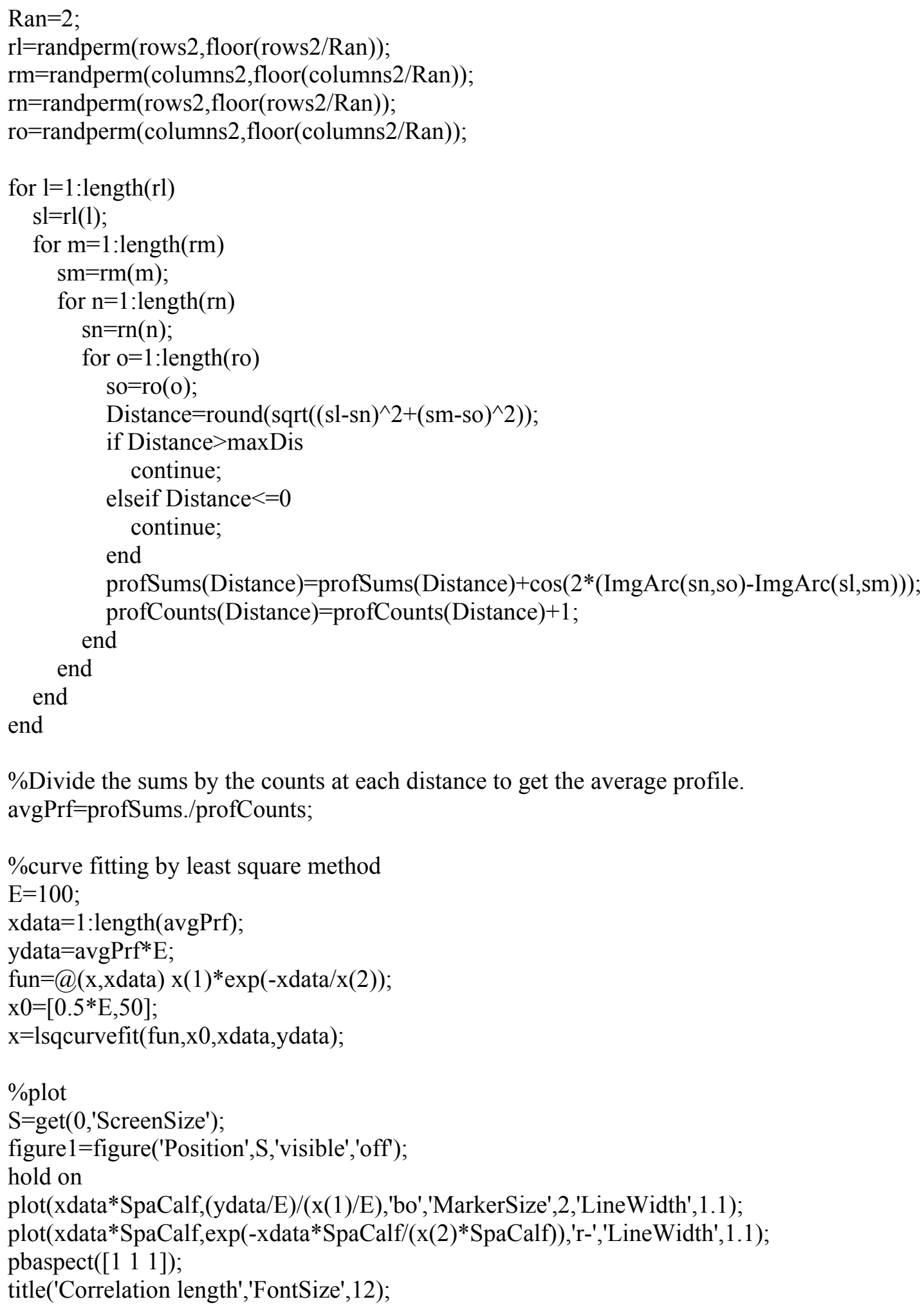

\%Divide the sums by the counts at each distance to get the average profile. avgPrf=profSums./profCounts;

\%curve fitting by least square method

$\mathrm{E}=100$;

xdata=1:length(avgPrf);

ydata $=\operatorname{avgPrf} * \mathrm{E}$;

fun=@(x,xdata) x(1)*exp(-xdata/x(2));

$\mathrm{x} 0=[0.5 * \mathrm{E}, 50]$;

$\mathrm{x}=$ lsqcurvefit(fun, $\mathrm{x} 0$, $\mathrm{xdata}, \mathrm{ydata})$;

$\%$ plot

$\mathrm{S}=\operatorname{get}(0$, 'ScreenSize');

figure 1=figure('Position',S,'visible','off');

hold on

plot(xdata*SpaCalf,(ydata/E)/(x(1)/E),'bo','MarkerSize',2,'LineWidth',1.1); plot(xdata*SpaCalf,exp(-xdata*SpaCalf/(x(2)*SpaCalf)),'r-','LineWidth',1.1);

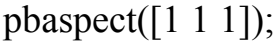
title('Correlation length','FontSize',12); 


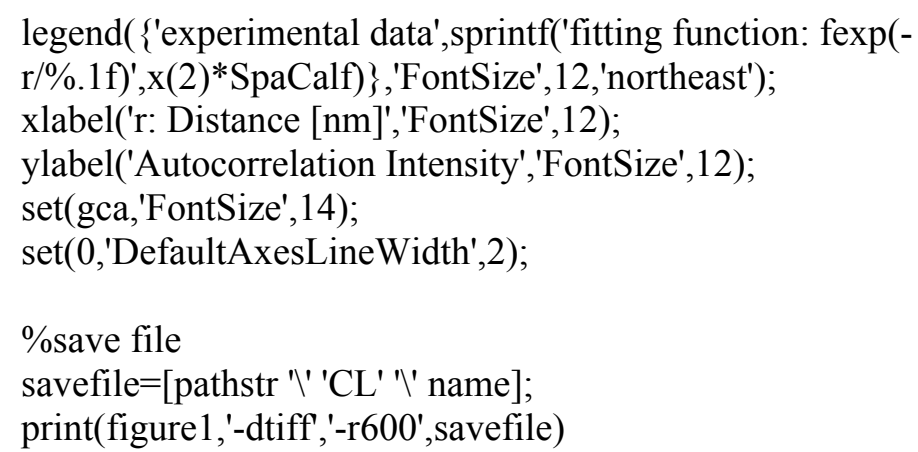

References

(1) Harrison, C. Ph.D. Thesis, Block Copolymer Microdomains in Thin Films. Princeton University, 1999.

(2) Ruiz, R.; Bosworth, J. K.; Black, C. T. Effect of Structural Anisotropy on the Coarsening Kinetics of Diblock Copolymer Striped Patterns. Phys. Rev. B 2008, 77, 054204. 